\title{
Agridoce: cozinhas profissionais pós-coloniais na pós-modernidade
}

\author{
Carlos Henrique Gonçalves Freitas ${ }^{1}$ \\ CÍNTIA RODRIGUES ${ }^{1}$ \\ VALDIR MACHAdO VALADÃO JUNIOR ${ }^{1}$
}

${ }^{1}$ Universidade Federal de Uberlândia (UFU) / FACUldAdE de Gestão e Negócios, UBerLÂNDIA - MG, BRASIL

\section{Resumo}

A desigualdade é uma questão histórica no Brasil, uma herança de injustiças sociais, econômicas, políticas e jurídicas emaranhadas e interdependentes. Este artigo é um resumo de uma abrangente pesquisa sobre cozinhas de restaurantes finos na cidade de Uberlândia, grande polo econômico e migratório na região central do Brasil, buscando expor instâncias de desigualdades replicadas em suas organizações. O presente trabalho faz um estudo crítico do desdobramento dos diálogos entre as perspectivas dos funcionários desses estabelecimentos, a partir de suas contextos socioculturais, e as perspectivas e particularidades das organizações, usando as noções de medievalidade, cidade global e paisagem alimentar como categorias de análise, com considerações adicionais em estudos de organização, pós-colonialismo e pósmodernidade. O material empírico do corpus de pesquisa foi coletado por meio do acompanhamento de chefs em dois desses restaurantes e, em seguida, analisado à luz dessas categorias e considerações. O trabalho revela que os funcionários, as organizações, e seus contextos reproduzem símbolos, comportamentos e representações que podem ser fontes de distinção social para seus clientes, mas, paradoxalmente, podem também reforçar as desigualdades que motivaram a pesquisa.

Palavras-chave: Sombreamento. Restaurante requintado. Foodscape. Pós-modernidade. Cidade global.

\section{Sweet \& sour: Postcolonial professional kitchens in the postmodernity}

\begin{abstract}
Inequality is a historical issue in Brazil, an inheritance of entangled and interdependent social, economic, political and legal injustices. This article summarizes a research on fine-dining restaurant kitchens in the city of Uberlandia, a major economic and migration hub in central Brazil, seeking to expose instances of inequalities replicated in these organizations. It attempts to offer a critical study of unfolding dialogues between its employees' perspectives of their socio-cultural contexts and those of the organizations and their own contextual particularities, using the notions of medievality, global city and foodscape as categories of analysis, with further considerations on organization studies, postcolonialism and postmodernity. Its research corpus' empirical material was collected through shadowing chefs in two restaurants and was analyzed in the light of those categories and considerations. It was possible to interpret that such workers, organizations, and their contexts reproduce symbols, behaviors and representations that may operate as sources of social distinction for their customers, but, paradoxically, may reinforce the inequalities that motivated the research.
\end{abstract}

Keywords: Shadowing. Fine-dining restaurant. Foodscape. Postmodernity. Global city.

\section{Agridulce: cocinas profesionales poscoloniales en la posmodernidad}

\section{Resumen}

La desigualdad es una cuestión histórica en Brasil, herencia de injusticias sociales, económicas, políticas y jurídicas enmarañadas e interdependientes. Este artículo es el resumen de una investigación exhaustiva sobre cocinas de restaurantes finos de la ciudad de Uberlândia -gran polo económico y migratorio de la región central de Brasil-, que se propone exponer instancias de desigualdad replicadas en sus organizaciones. El presente trabajo estudia críticamente el desdoblamiento de los diálogos entre las perspectivas de los empleados de dichos establecimientos, a partir de sus contextos socioculturales, y las perspectivas y particularidades de las organizaciones, usando las nociones de medievalidad, ciudad global y paisaje alimentario como categorías de análisis, con consideraciones adicionales en estudios de organización, poscolonialismo y posmodernidad. El material empírico del corpus de investigación se recolectó por medio del seguimiento de chefs en dos de esos restaurantes, y luego se analizó a la luz de esas categorías y consideraciones. El trabajo revela que los empleados, las organizaciones y sus contextos reproducen símbolos, comportamientos y representaciones que pueden ser fuentes de distinción social para sus clientes, pero, paradójicamente, también pueden reforzar las desigualdades que motivaron la investigación.

Palabras clave: Ensombrecimiento. Restaurante sofisticado. Foodscape. Posmodernidad. Ciudad global. 


\section{INTRODUÇÃO}

O contrastante sabor agridoce está presente em várias culinárias e pode abrir uma discussão para uma infinidade de influências sobre um tipo específico de organização: os restaurantes de alta gastronomia no Brasil. Este artigo procura discutir elementos de alguns desses contrastes, derivados de categorias de análise pós-coloniais e pós-modernas. Eles podem oferecer perspectivas igualmente contrastantes de contextos específicos e comuns em termos culturais, sociais, econômicos e políticos. Em um país marcado por um emaranhado de desigualdades econômicas, sociais, políticas e jurídicas interdependentes (COSTA, 2018), é razoável presumir que precedentes, circunstâncias, implicações e arranjos relacionados a naturezas contrastantes possam ser encontrados nos contextos sociais e de trabalho dessas organizações.

O restaurante de alta gastronomia pode ser definido por um conjunto de critérios: preços altos com base em seus alimentos e serviços, a criatividade de seus pratos e seu ambiente sofisticado (GAMBATO e GONÇALVES, 2017); influências estrangeiras presentes neles, especialmente francesas e italianas (BUENO, 2016); seus cuidados com a estética (IPIRANGA, LOPES e SOUZA, 2016); a organização e design de suas cozinhas à la carte (WALKER, 2014); e seus trabalhadores de cozinha, caracterizados por baixos salários, escolaridade, qualificação e experiência (COELHO e SAKOWSKI, 2014). Nesse setor, pode ser preferível contratar pessoas não qualificadas de origem social menos privilegiada e oferecer treinamento "in-service" (SUAUDEAU, 2004; FONSECA, 2013). Portanto, é provável que suas equipes de cozinha não estejam originalmente familiarizadas com os serviços e produtos que eles elaboram e com seus contextos de consumo, assim como seus trabalhadores podem não ter condições de pagar para comer no restaurante em que trabalham. Como os clientes esperam pratos, gostos e serviços diferenciados e elaborados, os funcionários da cozinha podem precisar de treinamento em novas habilidades, aclimatação com novos rituais e trabalhar com novos ingredientes e produtos (BOULUD, 2004; FONSECA, 2013; GAMBATO e GONÇALVES, 2017). $O$ contraste entre as expectativas dos clientes e as tradições dos cozinheiros pode criar possibilidades de distinção, bem como de construção, manutenção ou reforço de desigualdades que se estendem além da organização (MAGUIRE, 2016).

As noções de modernidade medieval e de cidades globais em países emergentes (ALSAYYAD e ROY, 2006) podem ajudar a contextualizar o restaurante de alta gastronomia, no Brasil. Esses autores argumentam que as cidades globais de hoje apresentam uma diversidade de enclaves urbanos e sociais com expectativas, comportamentos, valores e normas específicas, resultando em uma multiplicidade de sistemas ou jurisdições de governança - que por sua vez refletem realidades e interesses quase paralelos, muitas vezes contraditórios no mesmo espaço, como nas cidades medievais. Para eles, a medievalidade configuraria uma categoria de análise trans-histórica útil para entender cenários plurais contemporâneos, que de outra forma poderiam ser caracterizados por uma crescente compressão de espaço e tempo e antinomias pós-modernas, como em Harvey (1989) ou Jameson (1998); e pela fragmentação, ambiguidade, instabilidade, incerteza e fluidez da sociedade contemporânea, como em Judt (2010) ou Bauman (2012). Surge, então, um cenário favorável a igualmente múltiplas narrativas, valores, necessidades, desejos e interesses, bem como a agendas coletivas menos coesas e a políticas de identidade. Assim, a cozinha requintada de um restaurante pode abrigar tensões ou incongruências resultantes de um contexto paradoxalmente comprimido e fragmentado, global e múltiplo.

Partindo de Sassen (1991) - que cunhou o termo "cidade global" como um espaço de convergência de entidades subnacionais e supranacionais em um processo de integração e transnacionalização das etapas da produção econômica - Alsayyad e Roy (2006) expandem essa noção para discussões sobre jurisdição e cidadania à luz de fenômenos adjacentes, como desterritorialização, perda de raízes, deslocamento, fragmentação e guetificação, que também podem levar à perspectivas pós-coloniais. O ponto central de Alsayyad e Roy (2006) sobre cidades globais se distancia, nesse sentido, da perspectiva de Sassen (1991). Alsayyad e Roy (2006) enfocam a questão de múltiplas jurisdições que podem ser construídas, desenvolvidas e sustentadas nos espaços das cidades globais. No entanto, a noção inicial de Sassen (1991) não perde sua relevância como categoria de análise centrada em torno de uma infraestrutura econômica; ela e pensadores marxistas como Harvey (1989) e Jameson (1998) oferecem contribuições importantes para qualquer investigação em ciências sociais. Também não se deve ignorar a presença de considerações econômicas significativas em Alsayyad e Roy (2006). As múltiplas jurisdições das cidades globais implicam outros fenômenos significativos relacionados à economia, que juntos podem certamente ocorrer em relações próximas e mútuas.

Este trabalho propõe estender a ideia dos contrastes na modernidade medieval ao contexto organizacional e social de algumas cozinhas profissionais, um espaço de trabalho e uma extensão da sociedade que podem replicar tais compressões, fragmentações e multiplicidades paradoxais. Para obter seu material empírico, acompanhamos os chefs em dois restaurantes 
gastronômicos na cidade de Uberlândia, em um exercício de "shadowing". Essa é uma cidade de tamanho médio (cerca de 690.000 habitantes) e um grande centro regional, localizado no coração do Brasil, que tem experimentado um crescimento econômico acelerado, atraindo negócios e migrantes em busca de oportunidades e emprego nos últimos cinquenta anos (BERTOLUCCI, 2018). Processos acelerados de crescimento econômico e demográfico são geralmente acompanhados de repercussões sociais, econômicas e políticas que aumentam a fragmentação urbana e social em enclaves de interesses, agendas, expectativas, comportamentos, valores e normas múltiplas, como nas inchadas cidades globais de Alsayyad e Roy (2006).

\section{Paisagem Alimentar, Cultura e Política}

Apesar da relevância dos alimentos e da alimentação para a sobrevivência biológica (ROZIN, 2007); elas se tornaram essencialmente um fenômeno cultural, um conjunto de rituais sociais e historicamente construídos (HEGARTY e O'MAHONY, 2001), ou seja, noções culturais intangíveis. As escolhas, classificações e comportamentos relacionados a elas variam, então, de acordo com símbolos, representações e outras construções imaginárias que refletem visões de mundo e códigos sociais presentes na maneira como as pessoas interagem umas com as outras e com seu ambiente social e natural (MACIEL, 2004). Isso pode ser interpretado como um sistema simbólico relacionado à vida cultural de uma comunidade. Pode-se, então, definir a cozinha como o espaço do ritual dos alimentos e da alimentação por excelência e, talvez, um microcosmo da sociedade (DOS SANTOS, 2011). Portanto, uma cozinha profissional pode ser uma fonte legítima de perspectivas sobre relacionamentos relevantes, ricos, complexos, entrelaçados e múltiplos ou sobre o fluxo contínuo entre a organização e seu ambiente externo, onde conjuntos de arranjos, tradições, rituais, técnicas, ingredientes, pratos, artefatos e gostos específicos de lugares, regiões ou países ganham vida. Maciel (2004) vê a culinária como um sistema de alimentos e alimentação, enquanto Lévi-Strauss (1968) a vê como uma linguagem que inconscientemente traduz as estruturas e contradições - como na metáfora agridoce de uma sociedade, incluindo representações abstratas de arranjos sociais, econômicos e políticos, permitindo aproximar as noções de alimentos e alimentação e de cozinha e culinária à ideia de identidade (COLLAÇO, 2009; BUENO; 2016).

Alimentos, alimentação, cozinha e culinária juntos podem abranger ideias, práticas, comportamentos, preferências e artefatos que operam de maneira complementar, suplementar ou de ambas as formas; implicando múltiplas percepções e expressões do modo de vida de um grupo (MACIEL, 2004). Eles podem assumir a forma de etiquetas à mesa, de práticas e comportamentos ligados ao ritual social de compartilhamento de uma refeição (LINARES e TRINDADE, 2011); de padrões de consumo e gosto (DOS SANTOS, 2011); e de outras representações que extrapolam seu domínio imediato e permeiam relações abstratas, econômicas, sociais e políticas (DOS SANTOS, 2011), organizadas dentro de um grupo, em um determinado território e tempo, em uma concepção de cozinha (MACIEL, 2004). É possível vislumbrar, assim, a relação entre a culinária e a identidade de um grupo (FISCHLER, 1988; ROZIN, 2007; DE SOLIER, 2013). Também pode emergir o papel de uma cozinha no processo de identificação com instituições políticas, como um Estado-nação (BUENO, 2016). Tais noções do binômio alimentos e alimentação se aproximam de uma identidade nacional imaginada (ANDERSON, 1991; HOBSBAWM, 2000), e suas dimensões sociais e políticas podem ser identificadas como marcadores de capital social e distinção (BOURDIEU, 1984; ROZIN, 2007).

No entanto, essas noções podem ser consideradas sob uma única ótica: a da paisagem alimentar (traduzida do original "foodscape", em detrimento do termo paisagem gastronômica, buscando evidenciar um maior alcance do fenômeno, para além da imediata discussão valorativa de uma prática alimentar limitada a um certo gosto de consumo), ou uma paisagem de espaços e práticas culturais dinâmicas de alimentos e alimentação que são material e socialmente mediadas e construídas na sociedade por seus indivíduos, grupos e instituições (JOHNSTON e BAUMANN, 2010; JOHNSTON e GOODMAN, 2015). Essa paisagem também é uma arena na qual forças públicas, privadas, individuais, institucionais, sociais, políticas e econômicas interagem, refletindo quais alimentos são valorizados, produzidos, vendidos e consumidos; como e quais agentes podem influenciar ou controlar recursos e escolhas, individuais ou coletivas; e quem ou como esse alguém participa desse sistema, seja como consumidor, produtor, formador de opinião ou qualquer outro agente. Essa noção de paisagem alimentar reflete as relações dialéticas entre elementos culturais inter-relacionados socialmente construídos, incluindo artefatos e outras reificações, bem como suas dimensões políticas e econômicas (JOHNSTON e GOODMAN, 2015). Contudo, tal paisagem também pode ser a arena de processos não determinísticos, de maneira comparável a uma cosmologia de influências que resulta não necessariamente em uma perspectiva negativa de perda cultural, mas na transformação do objeto inicial ou na intensificação de alguns de seus traços definidores (SAHLINS, 1988).

Complementarmente, a maneira como os membros do grupo preparam sua comida pode indicar seu nível de civilização (LÉVI-STRAUSS, 1968), e o que e como as pessoas comem podem até ajudar a defini-las e entender seu ambiente social 
(ROZIN, 2007). A paisagem alimentar pode ser uma chave para identidades individuais e de grupo central a arranjos de poder em termos de identidade, identificação, distinção e política, à luz dos estudos pós-coloniais e do chamado processo de globalização (BOURDIEU, 1984; BUENO, 2016; WARDE, 2016). A cozinha de um grupo, portanto uma paisagem alimentar, reflete um conjunto de hábitos, práticas e preferências culinárias que podem revelar aspectos socioeconômicos, como padrões de consumo, produção, divisão e relações trabalhistas, distribuição de terras e riquezas e arranjos políticos.

Por outro lado, Warde (2016) indica que, embora a alimentação possa contribuir para a definição de uma identidade e de um modo de vida, o consumo, em qualquer paisagem alimentar, pode ser caracterizado pela falta de características e generalizações perceptíveis. Se, como qualquer outra expressão cultural, a paisagem alimentar pode representar um elemento de criação, inovação e continuidade das relações sociais que fortalecem a identidade coletiva e individual, também pode dar uma sensação de alteridade e exclusão em termos de classe, etnia, gênero ou nacionalidade (MINTZ e DU BOIS, 2002). Uma cozinha nacional pode materializar a identidade de grupo dentro de um dado tempo e território, reforçando o senso de pertencimento (MINTZ e DU BOIS, 2002; COLLAÇO, 2013), mas também pode ser um marcador de dominação cultural ou relações pós-coloniais (ZAMAN, SELIM e JOARDER, 2013). Também pode levar ao consumo de alimentos sem vínculos históricos ou culturais e desconectado das práticas socioculturais locais, descontextualizadas, sem contribuir como elemento de identificação coletiva (FISCHLER, 1988). Dada uma paisagem alimentar dinâmica e heterogênea, seus arranjos culturais e materiais podem ser marcados por dimensões econômicas e políticas desiguais (JOHNSTON e GOODMAN, 2015), e os trabalhadores da cozinha, como veremos, podem ser considerados à luz de subjetividades pós-modernas e pós-coloniais, devido à sua condição fragmentada, múltipla, deslocada e descentralizada como migrantes (HALL, 1992).

\section{Formação Nacional, Mídia e Gentrificação}

Geertz (2000) enfatizou que a construção de uma nação do terceiro mundo poderia estar sujeita a versões atualizadas ou reinterpretações do passado do colonizador, embora reconhecendo que suas elites poderiam ter suas próprias fontes locais ou transversais de inspiração, gerando novas visões de país e nação. Ao aceitar que a culinária de uma sociedade possa traduzir estruturas nas quais o grupo opere (LÉVI-STRAUSS, 1968), pode-se também aceitar que haja vínculos entre a paisagem alimentar do grupo e outras categorias sociais de análise, como migração, demografia, poder e classe, que não são estranhos à construção da nação em um contexto colonial.

Alimentos e alimentação são um fenômeno cultural que corresponde a um caminho histórico (HEGARTY e O'MAHONY, 2001). É constituído em uma paisagem alimentar específica de forças públicas, privadas, sociais, políticas e econômicas em interação, contribuindo e ocorrendo em múltiplas interações síncronas e assíncronas (JOHNSTON e GOODMAN, 2015). Segundo Wilson e Donnan (1998), nação e estado nem sempre se encaixam em noções antropológicas sobre cultura, porque muitas vezes os três nem sempre compartilham as ideias de integridade, unidade, linearidade e separação temporal e espacial. Seria perfeitamente aceitável ter várias culturas em um espaço e tempo social específico (GEERTZ, 1973). No entanto, Geertz (2000) admite estudar cultura e nação para entender estados pós-coloniais.

A formação nacional e a culinária nacional fazem parte de uma paisagem alimentar específica, com tradições e histórias que se desdobram em alguma forma de identidade (COLLAÇO, 2009; HOBSBAWM, 2000; BUENO, 2016). Seus processos de formação podem contribuir para interpretações sobre como diferentes grupos podem se reunir em uma dada sociedade, ou como vários conjuntos de tradições fragmentadas de comida e bebida podem produzir simultaneamente paisagens de alimentos paralelas ou convergentes, muitas vezes mal interpretadas como homogêneas. De fato, a paisagem alimentar do Brasil é rica e diversificada como seu povo, resultante de uma colonialidade próxima a de muitas sociedades sul-americanas (QUIJANO, 2000). No Brasil, uma variedade de tons etnológicos e uma diversidade de tradições culturais caracterizam um "novo povo", formado por imigrantes de todos os continentes, em circunstâncias igualmente diversas, favorecendo a diversidade, mas também tornando-o um caldeirão altamente poroso e dinâmico, apoiado em desigualdades econômicas e de arranjos e estruturas de poder (RIBEIRO, 2006).

No final do século XIX, no Brasil, a imigração europeia se intensificou na tentativa de substituir a força de trabalho escrava e "embranquecer" a população (ALVIM, 1999). Chegaram grandes números de portugueses, espanhóis, alemães, italianos e levantinos, principalmente cristãos (FAUSTO, 1999), seguidos também por imigrantes japoneses (SAKURAI, 1999), contribuindo para arranjos e estruturas de poder complexos, diversos e desiguais na sociedade em termos de classe, divisão do trabalho, participação política, rentismo e concentração do controle e poder dos meios de produção, com suas devidas implicações 
nas diferentes esferas da cultura da sociedade, incluindo em sua paisagem alimentar. Itens importantes de alimentos foram herdados de brasileiros nativos (MACIEL, 2004), dos Andes (FERNÁNDEZ-ARMESTO, 2004) ou da África (CÂMARA CASCUDO, 2004). As rotas comerciais portuguesas contribuíram para o intercâmbio de frutas e legumes entre continentes (MACIEL, 2004), mas essa migração de gostos, ingredientes, técnicas e tradições também foi ajustada de acordo com os contextos ambientais, culturais, sociais, econômicos e políticos locais (CÂMARA CASCUDO 2004). Bem mais adiante, por exemplo, restaurantes italianos populares, em São Paulo, tornaram-se uma expressão cultural imaginada significativa, ajustando práticas e produtos alimentares italianos ao gosto local (COLLAÇO, 2009). De uma perspectiva pós-colonial, esses encontros podem ser interpretados como negociações entre múltiplas identidades em diferentes dimensões e como reconstrução de representações e subjetividades culturais (HALL, 1996).

Collaço (2009) também mostra como, de 1950 a 1970, os restaurantes italianos em São Paulo representaram uma fonte de lazer para a emergente burguesia cosmopolita da cidade - Rodríguez-Ferrándiz (2014, p. 329) argumenta que "cultural consumption is distinguished by a voracity for the new and by a demand for distinction which is more pronounced than in other sectors of consumption". Esse uso da cultura já foi discutido em Adorno (2005) e Bourdieu (1984) em suas conceituações, respectivamente, de pseudoindividualização e de habitus, capital social e distinção. Posteriormente, isso se comunicaria com o crescimento do negócio de alimentos e alimentação e com restaurantes ganhando importância na dinâmica social e econômica (HENDERSON, 2011). Adicione-se a isso que, no Brasil, a noção de "classe média" seja intimamente associada ao acesso a formas de consumo, entretenimento e lazer que os brasileiros associam à Europa e à América do Norte (SALATA, 2015), reforçando as noções de capital cultural e classe (BOURDIEU, 1984) e de distinção por meio de alimentos e da alimentação (BUENO, 2016) à luz, particularmente, das noções de chef celebridade e de "gastronomia mediática".

Tal paisagem alimentar pode indicar a convergência de grupos bastante diferentes, de múltiplas, diversas perspectivas, expectativas e discursos. Essa paisagem alimentar, complexa e heterogênea, pode permitir interpretações sobre alimentos e alimentação que abrangem relações intrínsecas de poder e elementos socioeconômicos no estudo da colonialidade, no caso do Brasil. Esse mesmo cenário foi e tem sido marcado pela desigualdade, desde o passado colonial, e perpetuado por emaranhados econômicos, sociais, políticos e jurídicos (COSTA, 2018), bem como reforçado à medida que o negócio de alimentos e alimentação se expande em combinação com as mídias sociais e com a globalização de mercados e gostos, em um processo de gentrificação por meio de alimentos (FREITAS e MEDEIROS, 2018).

Com a globalização, a chegada de chefs franceses para trabalhar em hotéis e restaurantes do país, a estabilização de sua moeda e sua abertura a produtos estrangeiros são considerações igualmente importantes (ROCHA, 2016). Segue-se o surgimento de uma nova geração de jovens chefs brasileiros, principalmente os provenientes da classe média e treinados na França ou nos EUA. $O$ gosto dos brasileiros de alta renda também se tornaria cada vez mais influenciado pela expansão dos negócios de alimentos e alimentação, de programas de TV e de conteúdo de mídia estrangeiros divulgados localmente; bem como pelas experiências gastronômicas dos brasileiros ao viajar para o exterior (BUENO, 2016). Como resultado, as desigualdades da sociedade brasileira tendem a se refletir no cenário dos restaurantes de alta gastronomia, em que os clientes exigem ambientes, produtos e serviços, de alimentos e alimentação, estranhos à maioria dos trabalhadores que os elaboram ou executam (FREITAS e MEDEIROS, 2018).

A figura do chef como uma celebridade e os programas de TV sobre gastronomia tornaram-se quase um binômio como pão e manteiga, muitas vezes essencial para o sucesso de empreendimentos no setor, influenciando gostos, comportamento do consumidor, valor da marca e outros marcadores de distinção (HENDERSON, 2011; LENGYEL e GATLEY, 2016) O fenômeno de estrelas midiáticas e dos influenciadores digitais ligados à gastronomia podem acrescentar relevância ainda maior à ideia da vida moderna como uma coleção de espetáculos, nos quais toda experiência vivida pelas pessoas pode se transformar em algum tipo de ato encenado, como uma dessubjetivação ou ressubjetivação de si mesmo, ou como uma agência indesejada diante das forças do mercado e das técnicas estatais (DEBORD, 2006). Complementarmente, a lembrança da noção de biopolítica de Foucault implica subjetivação à medida que o agente se torna parte de um conjunto de tecnologias de controle (FOUCAULT, 2008), que não é imposto, mas abraçado por esse mesmo agente, que os internaliza sob a racionalidade da governamentalidade e as noções de capital humano e de uma disputa entre os sujeitos de interesse e de direito (FOUCAULT, 2008).

Ademais, por exemplo, quando o programa MasterChef da BBC foi franqueado e passou a ser exibido no Brasil, ele foi traduzido para outro ambiente público e cultural, para outra paisagem alimentar e ocorreram vários deslocamentos (FREITAS e MEDEIROS, 2018). De uma perspectiva pós-colonial, nos tempos contemporâneos - marcados pela pós-modernidade esse encontro cultural pode ser mais bem interpretado como uma negociação entre e por meio de múltiplas identidades 
deslocadas e fronteiras nacionais em diferentes dimensões, e como uma reconstrução de representações e subjetividades culturais (HALL, 1992, 1996). Lembrando Bauman (2012), pode-se dizer que questões de subjetividade, existência e poder permanecem em um mundo fluido, cheio de atos múltiplos, divergentes e simultâneos, de espetáculos fragmentados, operando como um vórtice debordiano.

Assim, há as duas perspectivas contrastantes, porém convergentes, para essa análise. Primeiro, uma pós-colonial, reencenando a tentativa de dominação ou imposição de discursos, por exemplo, ao transplantar programas de TV gastronômicos estrangeiros para o Brasil. Segundo, ao reconhecer visões relevantes e contemporâneas rotuladas como modernidade líquida, hipermodernidade ou pós-modernidade, e caracterizadas por noções como multiplicidade, narrativas, mídia, hiperconsumo, fragmentação, relativismo, instabilidade e incerteza em várias dimensões, mas essencialmente expostas por tipos particulares de discursos e conjuntos de práticas (FOUCAULT, 2008).

A perspectiva pós-colonial está intimamente relacionada à gastronomia mediática, representada pela crescente presença da gastronomia na mídia devido à exposição de chefs famosos, ao movimento "foodie" - um fenômeno resultante do consumo voraz de sabores exóticos e produtos e mídias relacionados à culinária (JOHNSTON e BAUMANN, 2010; HENDERSON, 2011; JOHNSTON e GOODMAN, 2015; LENGYEL e GATLEY, 2016) - e às suas influências na própria mídia, no consumo e no comportamento social. A gastronomia mediática e a comida são os dois lados da mesma moeda, contribuindo para um processo de gentrificação por meio dos alimentos e da alimentação (ZUKIN, LINDEMAN e HURSON, 2015).

Ainda, a multiplicidade da sociedade interage com as tradições em um processo de transformação. Embora as tradições impliquem resistência à mudança (HOBSBAWM, 2000), elas podem ser genuinamente construídas dentro da sociedade, por ela e para ela, ou inventadas pelos detentores do poder em nome de interesses próprios (ANDERSON 1991; HOBSBAWM 2000), por exemplo, do nacionalismo ou colonialismo. Por outro lado, pode-se supor que as transformações possam ser maiores quando as tradições estão menos enraizadas na sociedade ou encontram menor aderência popular ou legitimidade entre seus membros. Em termos pós-coloniais, as subjetividades coexistem em uma fronteira dinâmica ou em um estado de interdependência, caracterizado não por sujeitos bem definidos, mas por "mobility, uncertainty and multiplicity of the fact of the constant bordercrossing itself "(GROSSBERG, 1996, p. 92). Novidades e mudanças podem estar mais próximas de Hall (1996) do que de reivindicações de identidades locais ou de uma visão binária sobre a relação entre o colonizador e o pós-colonizado, como em Said (2003) e Spivak (1994). Esse pode ser o caso da paisagem alimentar dos restaurantes de alta gastronomia no Brasil.

Enquanto o termo "foodie" possa a vir a ter conotações positivas ou negativas (JOHNSTON e BAUMANN, 2010), nomear alguém como tal implica distinção entre quem possui conhecimento e gosto sobre gastronomia e quem não possui, próximo à questão de classe e distinção, como em Bourdieu (1984). Ainda, no Brasil, a orientação cultural para o novo (HOFSTEDE, 1994) pode ser acompanhada de nostalgia por algumas raízes da colonialidade, e restaurantes sofisticados podem explorá-la à medida que seus clientes busquem o consumo como distinção por meio de novidades, identificação ou inserção colonial herdada de discursos hegemônicos ocidentais, aumentando desigualdades históricas. Mas o consumo não precisa ser negativo; o consumo de alimentos pode ajudar na disseminação do conhecimento, já que os "foodies" e a gastronomia mediática atraem mais pessoas para paisagens alimentares mais ricas, diversificas e para valores e práticas alternativas (JOHNSTON e BAUMANN, 2010). Esse consumo pode implicar também um viés ético na direção de um consumo mais saudável e responsável (DE SOLIER, 2013).

Na medida em que símbolos, representações e discursos sobre alimentos e alimentação presentes nos rituais de consumo podem ser afetados pelas práticas dos "foodies" e pela gastronomia mediática, no Brasil, tais fontes de construção de tendências exercem localmente uma influência significativa semelhante às das antigas potências coloniais (FREITAS e MEDEIROS, 2018). Deve-se reconhecer que as paisagens alimentares podem refletir fenômenos sociais que trazem as noções de capital cultural e capital econômico para uma mesma arena, apesar do arcabouço teórico particular de cada uma. Enquanto o capital cultural indica relações múltiplas e amplas, o capital econômico tem o peso de um aparato historicamente infraestrutural e determinístico. $O$ primeiro caso está mais próximo de considerações pós-modernas, de Pierre Bourdieu a Michel Foucault e Zygmunt Bauman. O segundo é mais sensível a uma postura ética intrínseca aos estudos pós-coloniais, com fundamentos em pensadores que vão de Karl Marx a Edward Said, Kwame Anthony Appiah e Stuart Hall.

Assim, as noções de capital humano e de sujeitos de interesse e de direito (FOUCAULT, 2008) podem ser complementares àquelas de habitus, capital social e distinção (BOURDIEU, 1984), reforçando a importância de considerar-se em conjunto ação individual e agendas e esforços coletivos, em particular em um mundo assombrado pelo fracasso da modernidade e pela 
aporia entre preocupações públicas e privadas (BAUMAN, 2012). Ainda, a gastronomia mediática e a gentrificação podem levar à reformulação de símbolos, práticas e representações nas paisagens alimentares, à descentralização de identidades e ao deslocamento de pessoas, alcançando consumidores, a sociedade em geral e particularmente os trabalhadores de cozinhas de restaurantes gastronômicos. É possível o benefício de abordagens heterodoxas de estudo do encontro de hábitos, gostos e outros símbolos valorizados pelos formadores de opinião - que já desfrutam de privilégios e são capazes de determinar o que é uma fonte de distinção ou não - com aqueles dos trabalhadores que lhes atendem. Em um contexto difuso, o processo de gentrificação por meio dos alimentos e da alimentação pode reforçar as próprias desigualdades que motivaram a presente pesquisa.

\section{PROCEDIMENTOS METODOLÓGICOS}

Buscamos oferecer uma análise crítica e interpretações a partir de trocas reveladas entre restaurantes requintados, trabalhadores de suas cozinhas e suas diferentes perspectivas e contextos culturais e históricos, principalmente em termos de considerações pós-modernas e pós-coloniais. Na coleta do material empírico, utilizamos o "shadowing" (CZARNIAWSKA, 2007; MCDONALD e SIMPSON, 2014), acompanhando chefs, cozinheiros e seus ajudantes, em dois restaurantes refinados ("Montecarlo" e "Atelie Sofia"), em Uberlândia, por dois meses. Essa escolha foi baseada em uma pré-seleção de restaurantes gastronômicos daquela cidade (TRIPADVISOR, 2018), em trocas de pontos de vistas com "foodies" locais e em visitas a oito dos restaurantes pré-selecionados.

Inicialmente, nos concentramos no trabalho de seus chefes executivos e em como eles se comunicavam, organizavam, motivavam, guiavam, instruíam, corrigiam, ajudavam, dificultavam, questionavam, respondiam, delegavam, ignoravam e interagiam com sua brigada de cozinha, atendentes de salão e equipe de gerência, em suas rotinas diárias (QUINLAN, 2008). O material incluiu observações e testemunhos ad hoc desses chefs, seguidos de anotações. Não nos limitamos a um processo descritivo, abrangendo interações entre o observador, os observados e outras pessoas presentes no espaço estudado (VÁSQUEZ, BRUMMANS e GROLEAU, 2012; JOHNSON, 2014). Com o estabelecimento de relações de empatia e confiança relevantes (GOBO, 2008; QUINLAN, 2008), também incluímos observações, testemunhos e interações com os membros da brigada, incluindo comportamentos, ações e discursos sobre seu contexto social mais amplo dentro e fora da organização (VÁsQUEZ, BRUMMANS e GROLEAU, 2012). Além disso, foram tiradas fotos como parte do material empírico.

Atenção especial foi dada a considerações éticas e à adequada preparação do observador (JOHNSON, 2014), pois o "shadowing" envolve a negociação de espaços, sentimentos, ideias, pensamentos, privacidade e identidades, expostas a relações de indefinição, pertencimento, presença e visibilidade (QUINLAN, 2008). Outro aspecto cuidadosamente considerado foi o equilíbrio entre familiaridade e estranheza (GOBO, 2008; VÁSQUEZ, BRUMMANS e GROLEAU, 2012) devido à experiência prévia do observador como cozinheiro, chef e consultor de restaurantes, por dez anos, e sua potencial influência nas observações e análises.

O trabalho de campo totalizou 216 horas de contato, em 2 meses, gerando 309 fotos e 49 páginas de anotações de campo. Foram considerados cinco modos cognitivos de coleta de material empírico: ouvir, questionar, observar, ler e refletir (GOBO, 2008). As páginas do caderno de campo foram divididas em três colunas ("o que eu vejo"; "como eu sinto isso" e "inferências"), com base nas categorias de anotação de Gobo (2008): de observação, metodológicas, teóricas e emocionais. A primeira coluna correspondia à descrição de tarefas, símbolos, interações, locais, procedimentos, etc., registrando informações como escutadas ou observadas (por exemplo, registro linguístico, jargão, gíria etc.). A segunda foi reservada para considerações sobre sensações, emoções e reações. A terceira foi dedicada a possíveis vínculos entre as observações, nossa base teórica e metodológica, o contexto do restaurante e os contextos profissionais e sociais de sua equipe. Devido à falta de espaço livre em cozinhas à la carte, a seu tempo acelerado, a suas tarefas simultâneas e a suas rotinas intensas, entrevistas formais foram evitadas para não comprometer o fluxo de trabalho. Também foram pesquisadas anotações sobre menus, folhas de dados e sinais. Depoimentos "ad hoc" foram conduzidos como entrevistas discursivas, frequentemente com viés especulativo, nas quais os observados tinham liberdade para conduzi-las em seus próprios termos, objetivos, esquemas e linguagem (GOBO, 2008). 


\section{ANÁLISE DOS RESULTADOS}

Em Uberlândia, o TripAdvisor (2018) listava 79 pizzarias, 31 churrascarias, 6 "steakhouses", 12 sushis, 10 restaurantes de frutos do mar e inúmeros restaurantes de cozinhas nacionais (72 brasileiros, 11 mexicanos, 20 italianos, 5 chineses, 3 libaneses, 2 franceses, 1 alemão, 1 australiano, 1 indiano e 1 espanhol), sugerindo a incorporação, por sua população, de uma variedade de gostos e culinárias em seus hábitos de entretenimento. A cidade também vem recebendo um número crescente de imigrantes de várias regiões do Brasil e do mundo, há cinco décadas, devido ao seu desenvolvimento rápido e constante, tornando-se um importante centro socioeconômico (BERTOLUCCI, 2018). Os diferentes antecedentes e histórias pessoais e profissionais desses novos habitantes têm contribuído para uma diversidade de culturas, aspirações e discursos. Essa maior diversidade e expansão acelerada aproxima a cidade da noção de cidades globais de países emergentes em Alsayyad e Roy (2006), levando a interações dinâmicas entre elementos de regionalidade, internacionalização e desigualdade social. Se considerarmos aspectos presentes na fluidez de Bauman (2012), é possível admitir pressões sobre as tradições e tendências à formação de grupos que sejam sujeitos a interesses e expectativas múltiplos e fragmentados.

\section{Calor e Emoções}

Inicialmente, nosso foco foi o restaurante Montecarlo, localizado em um bairro de classe média alta e oferecendo adaptações locais de clássicos franceses e italianos ao paladar, ao gosto e ao perfil de seus clientes locais, respeitando suas referências regionais e oferecendo um bom custo-benefício e a chance de distinção por meio de alimentos e alimentação. Seu salão é confortável; seus atendentes vestem uniformes clássicos; e suas louças, copos e talheres são de alta qualidade, todos compatíveis com atributos importantes em um restaurante à la carte (AZEVEDO, MOURA e SOUKI, 2015). Atende 280 lugares e tem um serviço de entrega, produzindo até 1.600 pratos em um "bom domingo" - como explicado por seu chef de cozinha. A cozinha fica tão agitada, então, que não nos foi permitido adentrá-la aos domingos. Toda semana, sua brigada de cozinha processa $2.000 \mathrm{~kg}$ de carne, peixe e frutos do mar; produz 900 litros de molho bechamel e 600 litros de molho de tomate; e prepara $420 \mathrm{~kg}$ de arroz (cru).

De sua brigada de dezoito pessoas, apenas cinco nasceram em Uberlândia, nove vieram de estados do norte e nordeste do país, menos ricos; apenas um possuía ensino superior e nenhum tinha qualificação formal como cozinheiro, apenas treinamento em serviço. No entanto, o desafio que enfrentam - desenvolver não apenas técnicas, mas adquirir novos conhecimentos, habilidades e, talvez, gostos e comportamentos - ocorre em um ambiente de trabalho muito exigente. 0 turno da manhã pode ir das 06:00 às 18:00 horas, com um intervalo de uma hora às 10:00. Uma cozinha profissional é uma fonte de exaustão física e emocional: longas horas em pé; gritos constantes; desacordos; barulho permanente dos motores de grandes equipamentos; calor intenso de fornos, fogões, churrasqueiras e banhos-maria; tarefas simultâneas; e uma série interminável de movimentos sincronizados e repetitivos como ajoelhar-se, esticar-se e saltear frigideiras pesadas - quase como uma banda de jazz, para lembrar Hatch (1999).

O "shadowing" também impõe uma carga emocional, mas não notamos grandes desavenças na cozinha, entendendo que os observados poderiam ter relutado a se expor perante estranhos (EMERSON, FRETZ e SAW, 2011). Mas não abandonamos aquele desejável estado de dúvida útil à etnografia (GOBO, 2008) e confiamos na familiaridade (EMMEL e CLARK, 2011) para poder ler nas entrelinhas. Decidimos não aceitar o local de trabalho como um espaço livre de problemas, nem ignorar sua pressão psíquica, principalmente devido à rígida hierarquia da cozinha e à tradição de "bullying" (GIOUSMPASOGLOU, MARINAKOU e COOPER, 2018). Apesar dessas possíveis restrições, houve piadas mútuas, reclamações e eventuais insatisfações com desempenhos alheios. Isso também era agravado por longas horas, que, de alguma forma, levam os trabalhadores da cozinha a passar mais tempo entre si do que com suas famílias, tornando quase automática a socialização entre si também fora do trabalho, em seu pouco tempo livre, por exemplo, jogando futebol ou bebendo sua cerveja. Tudo isso contribuiu para evidenciar um ambiente de trabalho agitado, cheio de referências desconhecidas de uma brigada formada principalmente por migrantes (portanto, com características culturais específicas bem diferentes do ambiente, produtos, serviços e clientes da empresa, além de histórias pessoais de sacrifício em busca de uma vida melhor); e com rotinas de trabalho caracterizadas por uma grande carga de demandas, altas temperaturas e a necessidade de muita atenção aos detalhes. Portanto, eles trabalhavam sob pressão constante e frequentemente em seus limites emocionais. 
Não obstante, o encontro mais significativo que tivemos foi com Maria (pseudônimo). Ela vinha trabalhando no Montecarlo há cinco anos como a ajudante de cozinha, responsável por lavar todas as panelas, louças e talheres, mas ainda encontrava tempo para auxiliar seus colegas, recusando-se a aceitar uma promoção, apesar da insistência dos proprietários. Maria tem $1,57 \mathrm{~m}$ de altura, aproximadamente 40 anos de idade, três filhas e é divorciada, mas parece muito mais velha. Conversamos logo em nossa primeira semana de "shadowing", dividindo uma mesa durante o almoço das brigadas, quando ela puxou uma conversa sobre religião, revelando ter sido dependente de crack por sete anos, antes de entrar em uma igreja neopentecostal, começar no restaurante e mudar sua vida. Seus deveres são muito bem definidos e lhe garantem um considerável grau de independência e isolamento, proporcionando condições ideais para quem busca paz e segurança, apesar de todo esforço físico e intelectual envolvido em suas funções. Como ela explicou, ali, "ela poderia estar com Deus sem ser incomodada". No caso dela, a organização não era uma prisão, mas um remédio para sua alma.

Tudo isso deixou claro que para gerenciar um grupo desses é necessário mais do que treinamento técnico; é preciso ser capaz de compreender e respeitar contextos múltiplos e complexos, não raramente problemáticos. Eles podem representar potenciais desafios à gestão, na medida em que limitações ao planejamento, implementação e operação do negócio podem surgir de diferentes fontes de carga emocional, relacionadas ou não com o trabalho, afetando o desempenho em termos de produtos, serviços e, até mesmo, comunicação com o cliente.

\section{Cordialidade, Trabalho e Capital}

Relações familiares ou de amizades entre empregador e empregado eram um atributo notável na contratação de funcionários, tanto no Montecarlo quanto no Atelie Sofia, ambos negócios familiares. Neste último, a família sempre se fez presente durante o "mise-en-place" e o serviço, permitindo observar melhor como a autoridade e as responsabilidades eram atribuídas de acordo com esses laços pessoais. Seu chef de cozinha era um homem de quarenta anos que trabalhara como cozinheiro em Milão (Itália) e Manchester (Reino Unido) por 10 anos. Entretanto, em várias ocasiões, ele foi corrigido sobre tarefas e rotinas relacionadas à cozinha - típicas atribuições diretas de qualquer chef - por alguns de seus subordinados que tinham um vínculo pessoal com os proprietários, em seus nomes, sem nenhuma explicação adicional ou justificativa, influindo visivelmente na capacidade gerencial do chef. Não obstante, semelhantes usos de laços familiares e de amizade também foram observado no Montecarlo em vários momentos.

Essas inobservâncias de atributos técnicos na cadeia gerencial expõem o peso que as relações pessoais tendem a ter na sociedade brasileira, nos contextos empresarial, social e político. Enquanto Bresler (1997) oferece uma análise clássica do paralelo entre uma organização e cultura familiar; Sousa Filho (2008) destaca a influência do patrimonialismo em discursos no Brasil. Ambos os autores, em certa medida, apontam para a noção de cordialidade de Buarque de Holanda (1995), uma tentativa de tratar o peso das relações pessoais na sociedade brasileira. Por outro lado, Souza (2019) oferece uma crítica à noção de Buarque de Holanda, alertando para sua possível contribuição a uma ideologia de Estado. Entretanto, este artigo vê a noção de cordialidade como a forma que a sociedade brasileira encontrou para substituir a racionalidade - celebrada desde o iluminismo europeu até a modernidade, mas gestada de forma dessincronizada durante o percurso do Brasil da colônia à república - como base de sua vida social, empresarial e política. A noção de cordialidade estaria mais próxima da ideia de favor, em um contexto marcado pelo patrimonialismo, no qual elementos públicos foram historicamente confundidos com aqueles privados pelos detentores do poder (FAORO, 2001), sem limitá-la a ideologia de Estado, ao invés, alcançando também as visões de mundo dos indivíduos. O favor estabeleceu-se, então, como uma moeda de troca e mediação nas relações tanto na esfera pública quanto na privada (MONTEIRO, 1996).

Mumby e Stohl (1991) argumentam que o discurso reflete como as relações de poder são mantidas e reproduzidas nas organizações. De uma perspectiva diferente, Foucault (2008) coloca o discurso como um elemento fundamental na maneira como as novas tecnologias de controle substituem o antigo poder disciplinar. 0 discurso, então, pode ser internalizado como uma forma de adesão a uma determinada ordem. Nesse sentido, importa menos o que as palavras denotam, como expressão de um indivíduo, do que a forma como atos de fala moldam os discursos persuasivos do grupo (VAN DIJK, 2012). Podemos, então, aceitar que o discurso organizacional também é uma forma de controle. No caso do Atelie Sofia, podemos interpretar que as relações pessoais e a cordialidade não apenas fazem parte desse controle, mas também são percebidas por sua equipe como um sinal de recompensa pelo trabalho e dedicação à organização, com cujos proprietários, afinal, muitos deles mantêm relações pessoais. O ponto que tentamos enfatizar é que esses vínculos podem, então, ser nutridos em proveito da organização, mas em detrimento de relações profissionais e da remuneração adequada do trabalho. A cordialidade pode, 
então, como proposto acima, não se limitar a um aparato ideológico de controle do Estado e de sua elite, como visto por Souza (2019), mas ser incorporada a visões de mundo individuais - as quais, no entanto, podem contribuir para esse mesmo aparato.

Ademais, o resultado indireto do emaranhamento entre colonialidade, cordialidade, trabalho e recompensa, em uma organização brasileira como o Atelie Sofia, pode ser ilustrada pela decisão dos proprietários de pagar US\$19.000,00 por um forno combinado de última geração (um equipamento que combina métodos de cozinhar, grelhar e assar no forno), por outro lado, recusando-se a pagar um salário mensal acima de US\$540,00 para um chef de cozinha brasileiro - como apuramos durante o "shadowing". Esse equipamento é sofisticado e específico para grandes operações, como navios de cruzeiro e grandes hotéis, excedendo as necessidades do Atelie. Essa aquisição pode refletir, então, a visão dos proprietários de que seja melhor investir desnecessariamente em equipamentos de grandes dimensões do que "investir" na qualidade de sua força de trabalho, em seu capital humano. Tudo isso leva à exposição da maneira como o trabalho manual tem sido historicamente desvalorizado no Brasil. Schwarcz e Starling (2015) apontam como qualquer trabalho manual costumava ser feito por escravos, no Brasil, mesmo em pequenos negócios artesanais, com em uma sapataria, em que o sapateiro tornava-se um mero proprietário dos escravos e da mais-valia de seus esforços. Isso levou a uma associação depreciativa entre trabalho (manual) e condições sociais subalternas (CALDEIRA, 1995).

Souza (2019) argumenta corretamente que essa forma de rede de relacionamentos também pode ser encontrada em outras sociedades. Contudo, a noção de cordialidade aqui argumentada pode ser vista como um fenômeno local específico, da mesma maneira que o uso de "guanxi" na China (CHENTING e LITTLEFIELD, 2001). Não obstante, as origens da cordialidade podem atravessar o caminho das questões da colonialidade. Essa discussão pode, portanto, contribuir para evidenciar o peso da colonialidade que ainda permeia a sociedade brasileira e como um restaurante pode refletir aspectos do pós-colonialismo, apesar da "modernidade" de seus produtos e serviços.

\section{CONCLUSÃO}

Nosso primeiro esforço foi desenvolver uma base teórica em torno da metáfora de categorias contrastantes de análises. Essa alegoria procurou apresentar como um espaço organizacional, a cozinha de restaurantes refinados em Uberlândia poderia ajudar na identificação de símbolos, representações, relacionamentos e narrativas que trouxessem consigo diferentes aspectos de seu contexto social mais amplo. Reconhece-se que o espaço organizacional e seus contextos sociais interno e externo fazem parte do mesmo todo e que possa ser difícil separá-los. No entanto, o uso de espaços sociais delimitados nos permitiu abordar metodicamente uma infinidade de fatores e aspectos em jogo. Dada a natureza cultural dos fenômenos sociais definidos pelo binômio de alimentos e alimentação (ROZIN, 2007) e sua resultante natureza multidisciplinar (FISCHLER, 1988), não foi possível, nem desejado, fazê-lo separadamente, mas sim abordar objetivos e perguntas, transversalmente e frequentemente simultaneamente.

Aspectos de pós-modernidade e pós-colonialismo estão presentes concomitantemente nos diálogos que ocorrem entre os trabalhadores e seus contextos; nos contextos socioculturais e organizacionais dos restaurantes; na construção da nação brasileira e no processo de emergência das cidades globais; bem como nas respostas e interpretações dos trabalhadores sobre os hiatos sociais, econômicos e políticos presentes em seus contextos. Essa interação e sobreposição de categorias de análise constitui a própria essência da pós-modernidade. Elas tornam-se um tema central, ao menos indiretamente, em Alsayyad e Roy (2006), Judt (2010) e Bauman (2012), bem como em teóricos cuja contemporaneidade lhes levou a considerá-las sem negligenciar possíveis incompatibilidades entre noções de pós-modernidade e a genealogia (FOUCAULT, 2008) e a dialética (HARVEY, 1989; JAMESON, 1998), tampouco negligenciando essenciais visões tradicionais e legítimas do pós-colonialismo

Procuramos oferecer considerações críticas e interpretativas sobre os diálogos entre as organizações e sua equipe de cozinha, incluindo respostas e atitudes em relação a contextos organizacionais e sociais específicos e comuns. Portanto, tentamos olhar para essas trocas e posições de diferentes perspectivas culturais e históricas, particularmente aderindo a contrastantes categorias de análise pós-coloniais e pós-modernas. Pudemos ver como os proprietários do Montecarlo e do Atelie Sofia procuraram elementos estrangeiros e/ou sofisticados, como sequências de apresentação de pratos; ingredientes novos, exóticos ou valorizados; e estética em decorações de pratos e uniformes. Nós os interpretamos como fontes de capital social e distinção (BOURDIEU, 1984). No entanto, embora esses elementos de distinção possam fazer sentido para os proprietários, 
suas organizações e clientes; para seus trabalhadores, esses elementos podem ser formas de aprofundar as desigualdades a que estão sujeitos, parcialmente originadas e explicadas por estudos pós-coloniais, bem como pela multiplicidade pós-moderna.

Também consideramos como as interpretações pós-coloniais da relação entre noção de cordialidade, trabalho e capital podem representar um obstáculo para aquelas pessoas que só podem confiar em seu trabalho para prover para si e suas famílias; e como esses trabalhadores têm muito pouco acesso aos frutos de seu trabalho duro, realizado sob extrema pressão física e psicológica. Por outro lado, aspectos relacionados à pós-modernidade e cidades globais, como diversidade e multiplicidade (ALSAYYAD e ROY 2006), fragmentação (BAUMAN, 2012) e pressão de discursos econômicos (FOUCAULT, 2008), em vez de reduzir a importância de abordagens pós-coloniais para nossa análise, podem contribuir para a interpretação de muitas relações entre essas organizações, seus trabalhadores de cozinha e seu contexto. A questão não reside no diagnóstico, mas na aparente falta de tratamento ou remédio adequado diante do enfraquecimento de discursos e agendas coletivas (BAUMAN, 2012). A maioria dos trabalhadores da cozinha que acompanhamos está em uma posição social frágil: mal remunerada, pobremente educada, mal treinada, muito ocupada no trabalho para buscar melhores oportunidades e impedida de qualquer ação civil por causa de um contexto social instável, incerto, fragmentado e confuso, o que pode dificultar agendas coletivas e favorecer políticas de identidade (BAUMAN, 2012). Depois de longos turnos e condições de trabalho difíceis, pode ser difícil pensar em outras coisas além de esquecer ou descansar, tampouco encontrar tempo para aulas noturnas ou ação política.

Finalmente, em termos de limitação da pesquisa, primeiro, houve a questão da familiaridade do observador com o ambiente das cozinhas de restaurantes brasileiros refinados, devido à sua história profissional, que procuramos moderar por meio de fundamentação teórica etnográfica e treinamento antes do "shadowing", particularmente em relação ao comportamento de campo e a questões éticas. Segundo, há a ausência de uma base teórica disciplinar específica para o tópico e os objetos da pesquisa, dadas suas naturezas multi, trans ou interdisciplinares. Tentamos lidar com essa ampla natureza disciplinar recorrendo a autores, pesquisas e publicações de vários campos do conhecimento.

Quanto a sugestões de pesquisas futuras, encontramos uma lacuna de estudos qualitativos e quantitativos que acompanhem o paradeiro de ex-cozinheiros no Brasil. A rotatividade de pessoal parece bastante significativa. No entanto, não encontramos nenhum estudo que investigasse se esses trabalhadores, a maioria deles muito jovens, continuam na profissão, por quanto tempo fazem isso e em que capacidade; ou se eles mudam completamente sua trajetória profissional e em que direção. Ainda, como durante o "shadowing", observamos o uso frenético de smartphones pela equipe, em seus intervalos; pode valer a pena uma investigação que identifique que tipo de mídia social esses trabalhadores usam e como, bem como uma análise de seus discursos - por exemplo, eles parecem preferir aplicativos de namoro específicos, conteúdos de vídeo e mensagens de voz ou com uso de imagens a mensagens de textos.

\section{AGRADECIMENTOS}

O presente trabalho foi realizado com apoio da Coordenação de Aperfeiçoamento de Pessoal de Nível Superior (CAPES) Brasil - Código de Financiamento 001." 


\section{REFERÊNCIAS}

ADORNO, T. W. On popular music. In: FRITH, S. and GOODWIN, A. (Eds.). On record. London: Taylor and Francis, 2005.

ALSAYYAD, N.; ROY, A. Medieval modernity: On citizenship and urbanism in a global era. Space and Polity, v. 10, n. 1, p. 1-20, 2006.

ALVIM, Z. M. F. O Brasil italiano (1880-1920). In: FAUSTO, B. (Ed.). Fazer a América: A imigração em massa para a América Latina. São Paulo: EUDUSP, 1999.

ANDERSON, B. Imagined Communities: Reflection on the origin and spread of capitalism. London: Verso, 1991.

AZEVEDO, L. G. M. R. P. P., MOURA, L. R. C.; SOUKI, G. Q. Um estudo qualitativo dos atributos para a escolha de um restaurante. Revista Acadêmica São Marcos, v. 5, n. 1, p. 25-51, 2015.

BAUMAN, Z. Liquid Modernity. Cambridge: Polity Press, 2012.

BERTOLUCCI, L. Similaridades e diferenças demográficas em municípios polos. In: Dinâmica Socioeconômica de Municípios Selecionados, v. 1. Uberlândia: CEPES/IERI-UFU, 2018.

BOULUD, D. Conselhos a um Jovem chef. São Paulo: Anhembi Morumbi, 2004.

BOURDIEU, P. Distinction: A social critique of the judgement of taste Cambridge, MA: Harvard University Press, 1984.

BRESLER, R. Roupa surrada e o pai: Etnografia em uma marcenaria. In: PRESTES MOTTA, F. C.; CALDAS, M. Cultura Organizacional e Cultura Brasileira. São Paulo: Atlas, 1997.

BUARQUE DE HOLANDA, S. Raízes do Brasil. São Paulo: Companhia das Letras, 1995.

BUENO, M. L. Da gastronomia francesa à gastronomia global: Hibridismos e identidades inventadas. Caderno $\mathrm{CrH}$, v. 29, n. 78, p. 443-462, 2016.

CALDEIRA, J. Mauá: Empresário do Império. São Paulo: Companhia das Letras, 1995.

CÂMARA CASCUDO, L. História da Alimentação no Brasil. São Paulo: Global, 2004.

CHENTING S.; LITTLEFIELD, J. E. Entering Guanxi: A business ethical dilemma in mainland China? Journal of Business Ethics. v. 33, n. 3, p. 199-210, 2001.

COELHO, M. H. P.; SAKOWSKI, P. A. M. Perfil da mão de obra do turismo no Brasil nas atividades características do turismo e em ocupações. Brasília, DF: IPEA, 2014.

COLLAÇO, J. H. L. Sabores e Memórias: Cozinha italiana e construção identitária em São Paulo. 2009. Tese (Doutorado em Antropologia Social) - Faculdade de Filosofia, Letras e Ciências Humanas, Universidade de São Paulo, São Paulo, 2009.

COLLAÇO, J. L. H. Gastronomia: a trajetória de uma construção recente. Habitus, v. 11, n. 2, p. 203-222, 2013.

COSTA S. Entangled inequalities, state, and social policies in contemporary Brazil. In: YSTANES M.; STR ØNEN I. (Eds.). The Social Life of Economic Inequalities in Contemporary Latin America. Basingstoke: Palgrave Macmillan, 2018.
CZARNIAWSKA, B. Shadowing: And other techniques for doing fieldwork in modern societies. Malmö: Liber AB, 2007.

DE SOLIER, I. Making the self in a material world: Food and moralities of consumption. Cultural Studies Review, v. 19, n. 1, p. 9-27, 2013.

DEBORD, G. Society of the Spectacle. New York: Zone Books, 2006.

DOS SANTOS, C. R. A. A comida como lugar de história: As dimensões do gosto. História: Questões e Debates, v. 54, n. 1, p. 103-124, 2011.

EMERSON, R. M.; FRETZ, R. I.; SHAW, L. L. Writing Ethnographic Fieldnotes. Chicago: University of Chicago Press, 2011.

EMMEL, N.; CLARK, A. Learning to use visual methodologies in our research: A dialogue between two researchers. Forum Qualitative Sozialforschung, v. 12, n. 1, p. 1-11, 2011.

FAORO, R. Os Donos do Poder: Formação do patronato político brasileiro. Porto Alegre: Editora Globo, 2001.

FERNÁNDEZ-ARMESTO, F. Comida: uma história. Rio de Janeiro: Record, 2004.

FISCHLER, C. Food, self and identity. Social Science Information, v. 27, n. 2, p. 275-292, 1988.

FONSECA, S. A. Ingredientes da Aprendizagem Social: Um estudo na cozinha de um restaurante da grande São Paulo. 2013. Tese (Doutorado em Administração de Empresas) - Universidade Presbiteriana Mackenzie, São Paulo, 2013.

FOUCAULT, M. The Birth of Biopolitics: Lectures at the Collège de France, 1978-1979. New York: Picador, 2008

FREITAS, C. H. G.; MEDEIROS, C. R. O. The business of food and eating: The postcolonial foodscape of Masterchef Brazil, social deconstruction and individual resilience. In: LATIN AMERICAN AND EUROPEAN ORGANIZATION STUDIES CONFERENCE, 7., 2018, Buenos Aires. Proceedings... Bueno Aires: LAEMOS and IAE Business School, 2018.

GAMBATO, C.; GONÇALVES, R. B. Adaptação da cozinha francesa à cultura e ao paladar brasileiro. CULTUR, v. 11, v. 3, p. 58-86, 2017.

GEERTZ, C. The Interpretation of Cultures: Selected essays. New York: Basic Books, 1973.

GEERTZ, C. The world in pieces: Culture and politics at the end of the century. In: GEERTZ, C. Available Light: Anthropological reflections on philosophical topics. Princeton: Princeton University Press, 2000.

GIOUSMPASOGLOU, C.; MARINAKOU, E.; COOPER, J. Banter, bollockings and beatings: The occupational socialisation process in Michelinstarred kitchen brigades in Great Britain and Ireland. International Journal of Contemporary Hospitality Management, v. 30, n. 3, p. 1882-1902, 2018.

GOBO, G. Doing Ethnography. London: Sage Publications, 2008.

GROSSBERG, L. Identity and cultural studies - Is that all there is? In: HALL, S.; DU GAY, P. Questions of Cultural Identity. London: Sage, 1996.

HALL, S. The Question of Cultural Identity. In: HALL, S.; HELD, D.; MCGREW, T. (Eds.). Modernity and its Futures. Cambridge: Polity, 1992.

HALL, S. When was 'the post-colonial'? Thinking at the limit. In: HAMBER, I.; CURTI, L. The Post-Colonial Question: Common skies, divided horizons. Abingdon: Routledge, 1996. 
HARVEY, D. The Condition of Postmodernity: An enquiry into the origins of cultural change. Oxford: Blackwell, 1989.

HATCH, M. J. Exploring the empty spaces of organizing: How improvisational jazz helps redescribe organizational structure. Organization Studies, v. 20, n. 1, p. 75-100, 1999.

HEGARTY, J. A.; O'MAHONY, G. B. Gastronomy: A phenomenon of cultural expressionism and an aesthetic for living. International Journal of Hospitality Management, v. 20, n. 1, p. 3-13, 2001.

HENDERSON, J. C. Celebrity chefs: Expanding empires. British Food Journal, v. 113, n. 5, p. 613-624, 2011.

HOBSBAWM, E. Introduction: Inventing traditions. In: HOBSBAWM, E.; RANGER, T. (Eds.). The Invention of Tradition. Cambridge: CUP, 2000.

HOFSTEDE, G. The business of international business is culture. International Business Review, v .3, n. 1, p. I-14, 1994.

IPIRANGA, A. S. R.; LOPES, L. L. S.; SOUZA, E. M. A experiência estética nas práticas culinárias de uma organização gastronômica. Organizações e Sociedade, v. 23, n. 77, p. 191-210, 2016.

JAMESON, F. The antinomies of postmodernity. In: JAMESON, F. The Cultural Turn: Selected writings on the postmodern 1983-1998. London: Verso, 1998.

JOHNSON, B. Ethical issues in shadowing research. Qualitative Research in Organizations and Management, v. 9, n. 1, p. 21-40, 2014

JOHNSTON, J.; BAUMANN, S. Foodies: Democracy and distinction in the gourmet foodscape. London: Routledge, 2010.

JOHNSTON, J.; GOODMAN, M. K. Spectacular foodscapes: food celebrities and the politics of lifestyle mediation in an age of inequality. Food, Culture and Society, v. 18, n. 2, p. 205-222, 2015.

JUDT, T. III Fares the Land. New York: Penguin, 2010.

LENGYEL, A.; GATLEY, A. Celebrity chefs and the construction of taste: From über macho to culinary crusader. In: COUNCIL FOR HOSPITALITY MANAGEMENT EDUCATION RESEARCH, LEARNING AND TEACHING ANNUAL CONFERENCE, 2016, Belfast. Proceedings... Belfast: Ulster University, 2016.

LÉVI-STRAUSS, C. Petit traité d'ethnologie culinaire. In: LÉVI-STRAUSS, C. L'Origine des Manières de Table. Paris: Plon, 1968.

LINARES, N. L.; TRINDADE, E. Processos de movimentos de significados simbólicos no consumo alimentar. Pensamento e Realidade, v. 26, n. 2, p. 46-64, 2011.

MACIEL, M. E. Uma cozinha à brasileira. Revista Estudos Históricos, v. 1, n. 33, p. 26-39, 2004.

MAGUIRE, J. S. Introduction: Looking at food practices and taste across the class divide. Food, Culture e Society, v. 19, n. 1, p. 11-18, 2016.

MCDONALD, S.; SIMPSON, B. Shadowing research in organizations: The methodological debates. Qualitative Research in Organizations and Management, v. 9, n. 1, p. 3-20, 2014.

MINTZ, S. W.; DU BOIS, C. M. The anthropology of food and eating. Annual Review of Anthropology, v. 31, n. 1, p. 99-119, 2002.

MONTEIRO, P. M. O homem cordial e a democracia quase impossível: Caráter típico-ideal e significado político da cordialidade em Raízes do Brasil. Ciência e Trópicos, v. 24, n. 2, p. 333-357, 1996.
MUMBY, D. K.; STOHL, C. Power and discourse in organization studies: Absence and the dialectic of control. Discourse and Society, v. 2, n. 3, p. 313-332, 1991.

QUIJANO, A. Coloniality of power and eurocentrism in Latin America. International Sociology, v. 15, n. 2, p. 215-232, 2000.

QUINLAN, E. Conspicuous invisibility: Shadowing as a data collection strategy. Qualitative Inquiry, v. 14, n. 8, p. 1480-1499, 2008.

RIBEIRO, D. O Povo Brasileiro: A formação e o sentido do Brasil. São Paulo: Companhia das Letras, 2006.

ROCHA, K. A. A evolução do curso de gastronomia no Brasil. Comportamento, Cultura e Sociedade, v. 4, n. 2, p. 11-27, 2016.

RODRÍGUEZ-FERRÁNDIZ, R. Culture industries in a postindustrial age: Entertainment, leisure, creativity, design. Critical Studies in Media Communication, v. 31, n. 4, p. 327-341, 2014.

ROZIN, P. Food and eating. In: KITAYAMA, S.; COHEN, D. (Eds.). Handbook of Cultural Psychology. New York: Guilford, 2007.

SAHLINS, M. Cosmologies of capitalism: The Trans-Pacific sector of "the world system". The British Academy: Proceedings, v. 74, p. 1-51, 1988.

SAID, E. Orientalism. London: Penguin, 2003.

SAKURAI, C. Imigração japonesa para o Brasil: Um exemplo de imigração tutelada (1908-1941). In: FAUSTO, B. (Ed.). Fazer a América: A imigração em massa para a América Latina. São Paulo: EUDUSP, 1999.

SALATA, A. R. Quem é classe média no Brasil? Um estudo sobre identidades de classe. Dados - Revista de Ciências Sociais, v. 58, n. 1, p. 111-149, 2015.

SASSEN, S. The Global City: New York, London, Tokyo. Princeton: Princeton University Press, 1991.

SCHWARCZ, L. M.; STARLING, H. M. Brasil: uma biografia. São Paulo: Companhia das Letras, 2015.

SOUZA, J. A Elite do Atraso: Da escravidão a Bolsonaro. Rio de Janeiro: Sextante, 2019.

SOUZA FILHO, O. M. Discurso autoritário, discurso subserviente e discurso libertário na estrutura patrimonialista brasileira. In: I JORNADA INTERNACIONAL DE ESTUDOS DO DISCURSO. Anais... Maringá: Universidade Estadual de Maringá, 2008. Disponível em: < http://www.ple.uem.br/jied/trab2.html >. Acesso em: out. 25, 2018.

SPIVAK, G. C. Can the subaltern speak? In: WILLIAMS, P.; CHRISTMAN, L. (Eds.). Colonial Discourse and Post-Colonial Theory: A reader. New York: Columbia University, 1994.

SUAUDEAU, L. Cartas a um Jovem Chef. Rio de Janeiro: Elsevier, 2004.

TRIPADVISOR. Melhores Restaurantes: Uberlândia, MG, Brazil. 2018. Disponível em:<https://www.tripadvisor.com.br/Restaurants-g303400Uberlandia_State_of_Minas_Gerais.html>. Acesso em: 10 fev. 2018.

VAN DIJK, T. A. Structures of discourse and structures of power. In: ANDERSON, J. A. Communication Yearbook 12. Abingdom: Routledge, 2012.

VÁSQUEZ, C.; BRUMMANS, B. H. J. M.; GROLEAU, C. Notes from the field on organizational shadowing as framing. Qualitative Research in Organizations and Management, v. 7, n. 2, p. 144-165, 2012. 
WALKER, J. R. The Restaurant: From concept to operation. Hoboken: Wiley, 2014.

WARDE, A. The Practice of Eating. Cambridge: Polity, 2016.

WILSON, T. M.; DONNAN, H. Nation, state and identity at international borders. In: WILSON, T. M.; DONNAN, H. Border Identities: Nation and state at international frontiers. Cambridge: CUP, 1998.
ZAMAN, S., SELIM, N.; JOARDER, T. McDonaldization without a McDonald's. Food, Culture and Society, v. 16, n. 4, p. 551-568, 2013.

ZUKIN, S.; LINDEMAN, S.; HURSON, L. The omnivore's neighborhood? Online restaurant reviews, race, and gentrification. Journal of Consumer Culture, v. 17, n. 3, p. 459-479, 2015.

Carlos Henrique Gonçalves Freitas

ORCID: https://orcid.org/0000-0002-9536-3693

Mestre em Administração de Empresas pela Universidade Federal de Uberlândia; Mestre em Linguística Aplicada pela Cardiff University; Mestrando em Economia pela Vrije Universiteit Amsterdam (interrompido), Escola de Negócios e Economia, Holanda. E-mail: chg.freitas@gmail.com

Cíntia Rodrigues

ORCID: https://orcid.org/0000-0001-7999-9002

Doutor em Administração de Empresas pela Fundação Getulio Vargas (FGV EAESP); Professor Adjunto e Coordenador dos Programas de Doutorado e Mestrado da Faculdade de Administração e Negócios da Universidade Federal de Uberlândia, Uberlândia- MG, Brasil. E-mail: cintia@ufu.br

Valdir Machado Valadão Junior

ORCID: https://orcid.org/0000-0001-7930-8056

Professor Titular de Doutorado e Mestrado na Faculdade de Administração e Negócios da Universidade Federal de Uberlândia, Uberlândia- MG, Brasil. E-mail: valdirjr@ufu.br 